Notfall Rettungsmed 2016 $19: 623-624$

DOI 10.1007/s10049-016-0254-6

Online publiziert: 25 . November 2016

(c) Springer Medizin Verlag Berlin 2016

CrossMark

\author{
H. Marung' $\cdot$ H. Dormann ${ }^{2} \cdot$ M. Baubin ${ }^{3}$ \\ 'Institut für Rettungs- und Notfallmedizin, Kiel, Deutschland \\ ${ }^{2}$ Zentrale Notaufnahme Klinikum Fürth, Fürth, Deutschland \\ ${ }^{3}$ Univ-Klinik für Anästhesie und Intensivmedizin, Tirol-Kliniken/Medizinische Universität Innsbruck, \\ Innsbruck, Österreich
}

\title{
Kennzahlen in der Notfallmedizin
}

\author{
„Nur was wir messen, können wir \\ verbessern"
}

Liebe Kolleginnen, liebe Kollegen,

der zweite Teil der Überschrift kommt vielen von Ihnen als regelmäßige Leser von Notfall + Rettungsmedizin bekannt vor: eine der „10 Thesen für 10.000 Leben“ lautete genau so als Ergebnis der 1. Bad Boller Reanimationsgespräche [1]. Sie sollte ausdrücken, dass zum Erreichen des Ziels, viele Tausend zusätzliche Leben nach außerklinischem Kreislaufstillstand zu retten, viel mehr gebraucht wird als Teamarbeit, Medikamente oder Technik. Nur aus einer umfassenden Untersuchung der Reanimationsergebnisse an möglichst vielen Standorten und einem anschließenden Abgleich untereinander sehen wir, welche Maßnahmen an den Patienten und in welcher Qualität wirklich durchgeführt werden.

Die dabei erfassten Parameter werden auch als Kennzahlen bezeichnet. Hinter diesem scheinbar abstrakten Begriff verbirgt sich genau, was für die Bewertung der Qualität unserer notfallmedizinischen Versorgung unabdingbar ist: ein objektiver, messbarer Kriterienkatalog auf Basis der jeweilig gültigen Vorgaben zur Diagnostik und Therapie, seien es Leitlinien, Empfehlungen oder Verfahrensanweisungen. Damit ist auch klar, dass sich Kennzahlen überall dort zur Beurteilung der Qualität in der Notfallmedizin eignen, wo entsprechende Vorgaben existieren.

Sie dienen nicht nur dazu, unterschiedlichste Notfallsituationen vom Polytrauma über den ischämischen Schlaganfall bis hin zur Anaphylaxie zu untersuchen. Kennzahlen können zur
Analyse sämtlicher Schritte der Notfallversorgung eingesetzt werden: vom Eingang des Notrufs über Diagnostik und Therapie durch den Rettungsdienst bis hin zur Weiterversorgung in einer geeigneten Klinik.

\section{》) Kennzahlen können zur Analyse sämtlicher Schritte der Notfallversorgung eingesetzt werden}

Pionierarbeit auf dem Gebiet der Qualitätssicherung im Rettungsdienst leisten seit einigen Jahren Lohs et al. Sie beschreiben in ihrer Übersicht den Ansatz und den Nutzen der trägerübergreifenden Qualitätssicherung in BadenWürttemberg. Basis ihrer Auswertungen sind Qualitätsindikatoren, also Kennzahlen, die Aussagen über die Einhaltung der gültigen Vorgaben zulassen. Die Autoren weisen dabei darauf hin, dass eine Interpretation der Ergebnisse differenziert erfolgen muss, um keine voreiligen bzw. falschen Schlüsse zu ziehen, und dass die Übermittlung erfasster Daten das Qualitätsmanagement vor Ort in den einzelnen Rettungsdienstbereichen nicht ersetzen kann.

Dax et al. nehmen den Leser mit in einen Bereich, der im Hinblick auf die Analyse und Verbesserung der Qualität viel zu lange im Abseits stand: die Rettungsleitstelle. Auch hier steht das Bundesland Baden-Württemberg, aber auch Bayern im Fokus. Die Autoren formulieren dabei das Ziel ei- ner länderübergreifend einheitlichen Definition zur Abbildung der Leitstellenleistung. Damit wäre ein objektiver Vergleich auch mit Leitstellen in Schleswig-Holstein oder Sachsen möglich und warum nicht auch in Basel oder Tirol?

Bernar et al. skizzieren für eben dieses österreichische Bundesland die Ergebnisse des ersten Tiroler NEF-BenchmarkBerichts, also der standortübergreifenden Analyse der Versorgungsqualität im Notarztdienst. Ziel des Berichts war, die Eignung der verwendeten Kennzahlen zu überprüfen, aber in erster Linie, Abweichungen von Standards zu erkennen sowie den Benchmark-Bericht unter den Notarztstützpunkten zu kommunizieren. Wie Arbeiten z. B. aus dem Bereich der Versorgung nach Herzinfarkt [2] oder Schlaganfall [3] zeigen, kann durch derartiges, regelmäßiges Feedback an die Durchführenden - ob Rettungsfachpersonal, Notärzte oder Mitarbeiter in den Notaufnahmen - tatsächlich das Ziel erreicht werden, nicht nur ein Strohfeuer zu entzünden, sondern die Versorgungsqualität langfristig zu verbessern.

Die Notaufnahmen als weiteres zentrales Glied der Versorgungskette von Notfallpatienten werden neben den Rettungsdiensten auch von einer ansteigenden Zahl von Akut- und Notfallpatienten frequentiert. Mit einer breiten $\mathrm{Pa}$ lette von diagnostischen und therapeutischen Möglichkeiten gilt es, den zeitkritisch und schwer kranken Patienten zu identifizieren, zu behandeln und weiter zu distribuieren. Qualitätsindikatoren zu diesem Versorgungsauftrag sind derzeit 
in Deutschland quasi nicht existent und aus der internationalen Literatur auch nur marginal übertragbar. Da derzeit aber durch verschiedene Arbeitsgruppen der DGINA (Deutsche Gesellschaft interdisziplinäre Notfall- und Akutmedizin) und der Sektion Notaufnahmeprotokoll der DIVI (Deutsche Interdisziplinäre Vereinigung für Intensiv und Notfallmedizin) sehr vielversprechende Initiativen gestartet wurden, freut es uns einen ersten Einblick in dieser Heftausgabe geben zu können. Kulla et al. beschreiben in diesem mit Bundesmitteln geförderten Forschungsprojekt einen spannenden Ansatz, mit dessen Hilfe sie die Eignung von international beschriebenen Qualitätsindikatoren für diesen speziellen Bereich untersucht haben und geben einen Ausblick für weitere Entwicklungsmöglichkeiten.

Schuster et al. vertiefen den Blick in die Notaufnahme und lenken den Fokus auf (Zitat) „geriatrische Notfallpatienten als Hochrisikopatienten“. Sie verweisen dabei auf die hohe Zahl an Todesfällen und Wiederaufnahmen nach der Erstvorstellung und fordern, neben dem Wissen um die jeweiligen Krankheitsbilder, Kenntnisse der altersphysiologischen Veränderungen und der speziellen Bedürfnissen dieser stetig wachsenden Altersgruppe. Für diesen Versorgungsauftrag konnten mit GeriQ-ED erstmals weltweit Struktur-, Prozess- und Ergebnisindikatoren entwickelt werden.

Wir laden Sie, liebe Leserinnen und Leser, ein, sich mit der ganzen Bandbreite des Themas „Kennzahlen in der Notfallmedizin“ vertraut zu machen. Damit möchten wir dazu beitragen, das Qualitätsmanagement vom Ruf eines bürokratischen Monsters zu befreien, als das es vielfach missverstanden wird. Vor allem aber sollen die vorliegenden Arbeiten dazu dienen, dass wir in allen Bereichen weiter lernen, miteinander ins Gespräch kommen, um die Versorgung der Notfallpatienten ständig zu verbessern.

Mit besten kollegialen Grüßen,

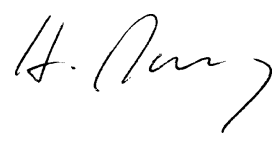

Hartwig Marung

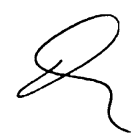

Harald Dormann

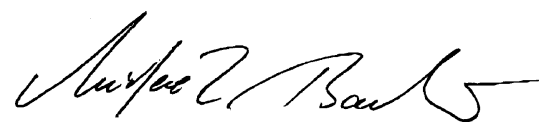

Michael Baubin

\section{Korrespondenzadresse}

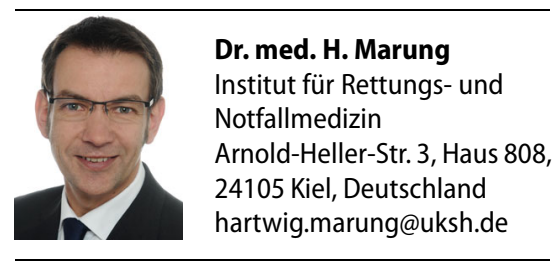

Interessenkonflikt. H. Marung, H. Dormann und M. Baubin geben an, dass kein Interessenkonflikt besteht.

\section{Literatur}

1. Müller MP, Kill C, Wnent J et al. (2014) Nur was wir messen können wir verbessern. NotfRettungsmed 17:325-326

2. Scholz KH, Hilgers R, Ahlersmann D et al (2008) Contact-to-balloon time and door-to-balloon time after initiation of a formalized data feedback in patients with acute ST-elevation myocardial infarction. Am J Cardiol 101:46-52

3. Ziegler V, Rashid A, Schaff M et al (2012) Qualitätsmanagement in der akuten Schlaganfallversorgung. Notarzt 28:237-245

\section{Diagnosen: Wann sind mehrere Ärzte besser?}

Kollektive Intelligenz ist ein vielversprechender Ansatz, um bessere ärztliche Entscheidungen zu treffen. Das konnten Studien des Max-Planck-Instituts für Bildungsforschung und des Leibniz-Instituts für Gewässerökologie und Binnenfischerei bereits zeigen. In einer Folgestudie untersuchten die Wissenschaftler nun, welche Voraussetzungen erfüllt sein müssen, damit die Gruppe erfolgreicher ist als der beste Einzelne in der Gruppe. Dabei zeigte sich, dass sich die Ärzte hinsichtlich ihrer Diagnosegenauigkeit ähneln müssen. Nur so können die kombinierten Entscheidungen mehrerer Ärzte die Entscheidung des besten Arztes der Gruppe überflügeln. Dieser Effekt zeigt sich auch bei verschiedenen Gruppengrößen oder unterschiedlichen Leistungsniveaus des besten Arztes innerhalb der Gruppe.

Für ihre Studie berechneten die Wissenschaftler anhand von über 20.000 Bewertungen von mehr als 140 Ärzten die Diagnosegenauigkeit der einzelnen Ärzte. Mit diesen Informationen simulierten sie, unter welchen Bedingungen die mittels Regeln der kollektiven Intelligenz kombinierten Diagnosen treffsicherer sind als Einzeldiagnosen. Das Ergebnis unterstreiche die Bedeutung der Diagnosegenauigkeit der einzelnen Entscheider für das Gesamtergebnis. Dies sollte auch in der Praxis berücksichtigt werden - beispielsweise bei der unabhängigen Doppelbefundung einer Mammografie-Aufnahme durch zwei Ärzte, so die Studienautoren.

Literatur: Kurvers RHJM, Herzog SM, Hertwig R et al (2016) Boosting medical diagnostics by pooling independent judgments. PNAS 8777-8782

Quelle: Max-Planck-Institut für Bildungsforschung www.mpib-berlin.mpg.de 